\title{
Radiative magnetohydrodynamic simulations of solar pores
}

\author{
R. Cameron ${ }^{1}$, M. Schüssler ${ }^{1}$, A. Vögler ${ }^{2}$, and V. Zakharov ${ }^{1}$ \\ 1 Max-Planck-Institut für Sonnensystemforschung Max-Planck-Str. 2, 37191 Katlenburg-Lindau, Germany \\ e-mail: [camerom; schuessler; zakharov] @mps .mpg.de \\ 2 Sterrekundig Instituut, Utrecht University, Postbus 80 000, 3508 TA Utrecht, The Netherlands \\ e-mail: A.Voegler@astro.uu.nl
}

Received 22 June 2007 / Accepted 23 July 2007

\section{ABSTRACT}

\begin{abstract}
Context. Solar pores represent a class of magnetic structures intermediate between small-scale magnetic flux concentrations in intergranular lanes and fully developed sunspots with penumbrae.

Aims. We study the structure, energetics, and internal dynamics of pore-like magnetic structures by means of exploratory numerical simulations.

Methods. The MURaM code has been used to carry out several 3D radiative MHD simulations for pores of various sizes and with different boundary conditions.

Results. The general properties of the simulated pores (morphology, continuum intensity, magnetic field geometry, surrounding flow pattern, mean height profiles of temperature, pressure, and density) are consistent with observational results. No indications for the formation of penumbral structure are found. The simulated pores decay by gradually shedding magnetic flux into the surrounding pattern of intergranular downflows ("turbulent erosion"). When viewed under an angle (corresponding to observations outside solar disc center), granules behind the pore appear brightened.

Conclusions. Radiative MHD simulations capture many observed properties of solar pores.
\end{abstract}

Key words. Sun: magnetic fields - Sun: photosphere - Sun: activity - Sun: magnetohydrodynamics (MHD)

\section{Introduction}

Solar pores are defined by Bray \& Loughhead (1964) as "simply small sunspots with no penumbral structure" or as "naked umbrae" by Solanki (2003). They have diameters of a few Mm at the visible solar surface and a lifetime of typically less than a day (Keppens 2000). The absence of a penumbra suggests that pores may have a rather simple magnetic structure, which could be represented by a magnetostatic flux tube with a predominantly vertical magnetic field (e.g., Simon \& Weiss 1970; Pizzo 1986).

Bray \& Loughhead (1964) already pointed out that pores are clearly distinguishable from dark elements in the granulation pattern through their much lower brightness and longer lifetime. Many of the more transient darkenings probably indicate shortlived magnetic features called micropores (Zirin \& Wang 1992), magnetic knots (Beckers \& Schröter 1968), or azimuth centers (Keppens \& Martinez Pillet 1996). Such concentrations of magnetic flux can appear as early stages of pore and sunspot formation; outside regions of flux emergence, however, they probably form accidentally as magneto-convective structures and dissolve within a few granule lifetimes (Bercik 2002; Bercik et al. 2003; Vögler et al. 2005).

The apparent simplicity of pores, notwithstanding the indications for substructure in the form of bright dots observed in large pores (e.g., Sobotka et al. 1999), and their position as an intermediate structure between the transient small-scale flux concentrations in the intergranular downflow network and full-fledged, long-lived sunspots make pores an attractive topic for a theoretical study. This is the more timely since 1) during the last decade a considerable body of high-resolution observations of good quality has been collected (e.g., Keppens \& Martinez Pillet 1996; Leka \& Skumanich 1998; Sütterlin 1998; Keil et al. 1999;
Sobotka et al. 1999; Dorotovič et al. 2002; Hirzberger 2003; Sankarasubramanian \& Rimmele 2003; Lites et al. 2004), and 2) pore-sized structures are now within reach of realistic radiative MHD simulations, while the few previous theoretical studies of pores were restricted to idealized potential-field models (Simon \& Weiss 1970; Simon et al. 1983), magnetostatic models (Pizzo 1986, 1990), and simplified 2D simulations (Knölker \& Schüssler 1988).

Here we report results from exploratory simulations of pores in the solar photosphere and uppermost convection zone carried out with the MURaM code (Vögler 2003; Vögler et al. 2005). We particularly address the stability and lifetime of pores, their thermal and magnetic structure, and their interaction with the surrounding granulation. Furthermore, we compare our simulation results with some observationally inferred properties of solar pores.

\section{Numerical model}

Pores are observed nearly exclusively in young active regions and in areas of magnetic flux emergence. This suggests that they are not an accidental phenomenon of surface magnetoconvection but probably represent rising and emerging magnetic flux bundles from the convection zone, or fragments thereof. This is consistent with the fact that pores do not form spontaneously in realistic magneto-convection simulations of quiet, network, or plage regions, while micropores come and go frequently (Bercik 2002; Vögler 2004b; Vögler et al. 2005). Consequently, unless the whole process of rise and emergence of a fragmented flux bundle is included, the simulation has to start with a pre-existing pore, represented by a plug of vertical magnetic flux as initial condition. This procedure has the 
advantage that the total magnetic flux of the pore can be freely chosen, so that the size-dependence of the various properties of pores can be studied.

\subsection{MURaM code}

The MURaM (MPS/University of Chicago RAdiative MHD) code has been developed jointly by the MHD simulation groups at MPS/Katlenburg-Lindau and at the University of Chicago (Vögler et al. 2003, 2005; Vögler 2003). The code treats the three-dimensional, time-dependent MHD equations for a compressible and partially ionized plasma and includes non-grey radiative energy transport based on the short-characteristics scheme (Kunasz \& Auer 1988) and opacity binning (Nordlund 1982; Vögler et al. 2004). The equation of state is determined including the ionization equilibrium for the first ionization of the 11 most abundant elements. The spatial derivatives are discretized with 4th-order centered differences on a threedimensional Cartesian grid; the time stepping is explicit with a 4th-order Runge-Kutta solver. The scheme is stabilized by the application of artificial diffusivities (shock-resolving diffusion and hyperdiffusivity) following Caunt \& Korpi (2001).

The MURaM code has been successfully used to simulate the structure and dynamics of (sub)photospheric magnetic flux concentrations and micropores (Vögler \& Schüssler 2003; Vögler et al. 2005) in comparison with spectro-polarimetric observations (Khomenko et al. 2005a,b; Shelyag et al. 2007), to examine the effects of non-grey radiative transfer on magnetoconvection simulations (Vögler 2004a), to study the physical origin facular brightening (Keller et al. 2004) and its effect on solar irradiance variations (Vögler 2005), to investigate the relationship between G-band bright points and magnetic flux concentrations (Schüssler et al. 2003; Shelyag et al. 2004), to analyse the reversed granulation phenomenon (Cheung et al. 2007b), to study the emergence of magnetic flux tubes (Cheung 2006; Cheung et al. 2007a), to analyse magneto-convection in sunspot umbrae (Schüssler \& Vögler 2006), and to study solar surface dynamo action (Vögler \& Schüssler 2007).

\subsection{Boundary conditions}

In the simulations presented here, the magnetic structure (pore) is embedded in a sufficiently large volume so that periodic boundary conditions at the vertical side boundaries can be assumed. The bottom boundary is treated as in previous MURaM simulations (open boundary, vertical magnetic field, regulation of mass and energy flux) except for the inflows (upflows) below the pore: whenever the magnetic field strength at the bottom exceeds $180 \mathrm{G}$, the temperature of the inflowing gas is reduced by a percentage, $q$, from the value determined by the algorithm regulating the total energy flux through the system. This procedure mimicks the inhibition of the convective energy transport by the magnetic field, which is consistently treated in the interior of the box, but for the inflows has to be prescribed through the boundary condition. It turns out that a long-lived (decay time of the order of an hour or more) and dark (bolometric brightness $\lesssim 40 \%$ of the quiet-Sun value) pore requires $q \gtrsim 7.5 \%$, which leads to an strong reduction of the inflow into the pore through the bottom of the computational box. Choosing larger values of $q$ does not significantly affect the properties of the simulated pores.

The top boundary of the simulation box is closed for flows. For the magnetic field, we have alternatively used two different boundary conditions: 1) vertical field or 2) matching to a potential field. Both conditions lead to stable pore structures, the vertical field case showing a somewhat slower decay, i.e., a smaller rate of flux loss to the surrounding network of intergranular lanes, particular in the case of a small pore (Cameron et al. 2004). There is no clear justification for assuming a vertical field boundary condition in the case of a pore; the potential field condition seems to be somewhat more realistic, particularly for an "older" pore in a magnetically relaxed environment. In any case, the simulation results show that the general evolution of the pore and its structure in the visible layers and below are not strongly affected by the choice of the upper boundary condition.

\subsection{Initial state}

Since pores do not form spontaneously from surface flux, we have constructed initial configurations with an imposed porelike magnetic flux concentration. In order to approach a thermally relaxed configuration, we first introduced a magnetic flux sheet into a statistically stationary $2 \mathrm{D}$ convection model and let the system evolve for 10-15 h (solar) time, during which the flux sheet remained mostly coherent. Thereafter, the 2D plane was cut along a line near the center of the flux concentration (where the field vector is approximately vertical) and swept out azimuthally to form an axisymmetric, 3D configuration in the rectangular simulation volume, applying the appropriate corrections to satisfy $\nabla \cdot \boldsymbol{B}=0$. The Cartesian geometry of the box and small perturbations break the azimuthal symmetry of the magnetic structure and the model was then evolved in $3 \mathrm{D}$ for about an hour of solar time. The resulting configurations were taken as the initial states of the pore simulations proper.

During the simulations, very low values of the plasma $\beta$ (ratio of gas pressure to magnetic pressure) appear occasionally in the upper part of the simulation box. To avoid exceedingly small timesteps in our (explicit) time integration, we have imposed a minimum value for the density of $5 \times 10^{-10} \mathrm{~g} \mathrm{~cm}^{-3}$ (a factor 10 smaller for the runs with potential-field boundary condition) and also a minimum internal energy density (per unit mass) of $2 \times$ $10^{11} \mathrm{erg} \mathrm{g}^{-1}$. This procedure only affects the layers of the simulated pore near the upper boundary and has almost no effect on the total mass and energy in the computational box.

\subsection{Simulation runs}

The parameters of the simulation runs carried out in the course of this study are summarized in Table 1 . The cases shown here have been run with grey radiative transfer. This simplification affects mainly the temperature fluctuations in the upper layers of the model (Vögler 2004a; Cameron et al. 2007), which are not relevant here, since we do not consider synthetic spectroscopic diagnostics in this exploratory study.

Run SV1, the simulation of a small pore with no reduction of the inflow temperature below the magnetic structure, results in the rapid dissolution of the pore before any significant darkening occurs at the surface. The effective suppression of the inflow below the pore by a sufficiently large value of $q$ leads to coherent dark magnetic structures. Some properties of run SV2 have been discussed in Cameron et al. (2004), so that we will concentrate here on the simulations of the larger pore. Most results shown in this paper are from run LV1.

The (explicit) magnetic diffusivity, $\eta$, has been chosen to be as small as possible while still keeping all magnetic structures resolved for a given grid spacing. Therefore, the simulations of small pores $(\mathrm{SV} 1 / 2, \Delta x=21 \mathrm{~km})$ have been carried out with 
Table 1. Parameters of the simulations runs. $\Phi_{0}$ : initial magnetic flux of the pore; $q$ : temperature reduction of the inflow at the bottom; $\Delta x$ : horizontal size of a grid cell; top b.c.: condition for the magnetic field at the top boundary. All simulations have been carried out using $288 \times$ $288 \times 100$ grid cells and a vertical cell size of $14 \mathrm{~km}$. The computational box for all "L" runs has a size of $12 \times 12 \times 1.4 \mathrm{Mm}^{3}$ while the "S" runs have a box of $\left(6 \times 6 \times 1.4 \mathrm{Mm}^{3}\right)$. The simulation runs cover between 45 and 60 min solar time, starting from the initial configuration described in Sect. 2.3

\begin{tabular}{c|ccccl}
\hline \hline Case & $\Phi_{0}\left[10^{20} \mathrm{Mx}\right]$ & $q[\%]$ & $\Delta x[\mathrm{~km}]$ & Top b.c. & Remark \\
\hline SV1 & 0.23 & 0 & 21 & vertical field & bright pore, dissolves rapidly \\
SV2 & 0.23 & 15 & 21 & vertical field & small pore, decays slowly \\
LV1 & 2.3 & 15 & 42 & vertical field & large pore, decays very slowly \\
LV2 & 2.3 & 7.5 & 42 & vertical field & test effect of smaller $q$ \\
LP1 & 2.3 & 15 & 42 & potential field & results similar to run LV1 \\
LP2 & 2.3 & 15 & 42 & potential field & $\sim 30 \%$ opposite-polarity flux outside pore \\
\hline
\end{tabular}

$\eta=1.1 \times 10^{11} \mathrm{~cm}^{2} \mathrm{~s}^{-1}$, while for the large pores $(\Delta x=42 \mathrm{~km})$ we have taken a value of $\eta=3.3 \times 10^{11} \mathrm{~cm}^{2} \mathrm{~s}^{-1}$.

In run LP2, some opposite-polarity magnetic flux (about $30 \%$ of the initial flux of the pore) was inserted in the exterior of the pore in order to test whether this would facilitate the formation of horizontal (penumbra-like) magnetic filaments. No such structures appeared and the presence of this flux did not affect the properties and evolution of the pore in any significant way.

Run LV2 has been carried out to evaluate the effect of reducing the parameter $q$, describing the temperature reduction in the upflows below the pore. The results turned out to be very similar to those of runs LV1 and LP1. There is very little inflow from the bottom into the pore in all cases with $q \geq 7.5 \%$.

\section{Results}

\subsection{Temporal evolution}

All simulated pores decay in time, i.e., the size of the dark area decreases and the magnetic flux gradually disperses into the downflow lanes of the surrounding granulation pattern. This process is illustrated in Fig. 1, which shows three snapshots of brightness and magnetic field of the pore simulated in run LV1 during about $1 \mathrm{~h}$ of solar time. Owing to the suppression of convective energy transport by the strong magnetic field, the pore clearly stands out as dark region in the brightness maps. The edges of the surrounding granules show a brightening with a contrast of about $5 \%$ over a width of $\sim 100 \mathrm{~km}$. The pore strongly flares out with height around optical depth unity, leading to a kind of "hot-wall effect" (Spruit 1976). A qualitatively similar effect was also found in two-dimensional models of pore-like flux concentrations (Knölker \& Schüssler 1988). The width of the bright rim is determined by radiative transfer effects and by the magnetic geometry of the pore. Viscous and resistive heating effects are insignificant for its structure, as demonstrated by the fact that its width is the same for all simulated pores, irrespective of the grid resolution and independent of the values for the viscous and magnetic diffusivities.

While the horizontal flows of the adjacent granules tend to keep the magnetic flux together, the simulated pore is also connected to downflow lanes into which magnetic flux gradually leaks out and becomes dispersed in the network of intergranular lanes. This erosion process (cf. Petrovay \& Moreno-Insertis 1997) apparently is the dominant mechanism by which the pore decays and eventually vanishes as a dark structure. Its magnetic flux remains in the system in the form of intergranular field. The continuous leakage of magnetic flux from the dark pore and its transport into the surrounding downflow network explains why the extension of the observed magnetic signal associated with a pore, the "magnetic radius", is found to be considerably larger than the dark structure, the "brightness radius" (Keppens \& Martinez Pillet 1996; Sütterlin et al. 1996; Keil et al. 1999). An interpretation along these lines was previously suggested by Martinez Pillet (1997, see his Fig. 3).

Figure 2 shows the time evolution of the magnetic flux contained in the simulated pores (runs LV1 and LP2). Here the pore is defined as the region with a brightness below $70 \%$ of the average brightness of the computational domain. Apart from the fact that the case LP2 has lost somewhat more flux in the initial relaxation phase (not shown in the figure) and shows somewhat more scatter, the decay is very similar in both cases. The fluctuations result from the intensity-based pore definition: areas at its periphery occasionally become brighter than the threshold and thus temporarily do not contribute to the total flux.

In order to study whether the decay mode of a pore could possibly be detected by considering the evolution of its total magnetic flux alone, we have drawn fit curves for run LV1 in Fig. 2: the full line corresponds to an exponential decay (volume diffusion) while the dashed curve represents a decay rate proportional to the length of the pore's circumference, thus representing "turbulent erosion". While we have seen above that the latter process is the most plausible description for the pore decay, both curves fit the points equally well, so that, owing to the small size and short lifetime of the pore, the mechanism cannot be uniquely determined from the flux decay alone. The characteristic exponential decay time of about 40 min corresponds to an effective ("turbulent") volume diffusivity of about $1.4 \times 10^{12} \mathrm{~cm}^{2} \mathrm{~s}^{-1}$, which is roughly a factor 4 larger than the assumed diffusivity parameter in the simulation. Interestingly, simulations of the decay of mixed-polarity fields in granular convection (Vögler et al., in preparation) with much higher resolution (and correspondingly smaller diffusivity parameters) give almost the same value for the "turbulent" diffusivity as found here. In turn, this value is within a factor of 2 of the simplistic estimate $u \cdot l / 3$ on the basis of the typical granular velocity, $u \simeq 1 \mathrm{~km} \mathrm{~s}^{-1}$, and size, $l \simeq 1 \mathrm{Mm}$.

The relationship between the total magnetic flux and mean bolometric disc-center brightness of the pore for various runs is shown in Fig. 3. There is a systematic trend: smaller pores are brighter and, accordingly, as a pore loses flux and shrinks in the course of its decay, its brightness increases. This effect is probably due to the growing effect of lateral radiative heating when the pore becomes smaller, the effect being enhanced by its increasingly non-circular, "ragged" shape.

Table 2 gives the mean continuum intensities for the three snapshots shown in Fig. 1 in a number of wavelengths that have been used in observational studies of pores. The size-dependence of the brightness is in qualitative agreement with various observations (e.g., Bonet et al. 1995; Keppens \& Martinez Pillet 1996; Mathew et al. 2007). In the visible, the absolute 

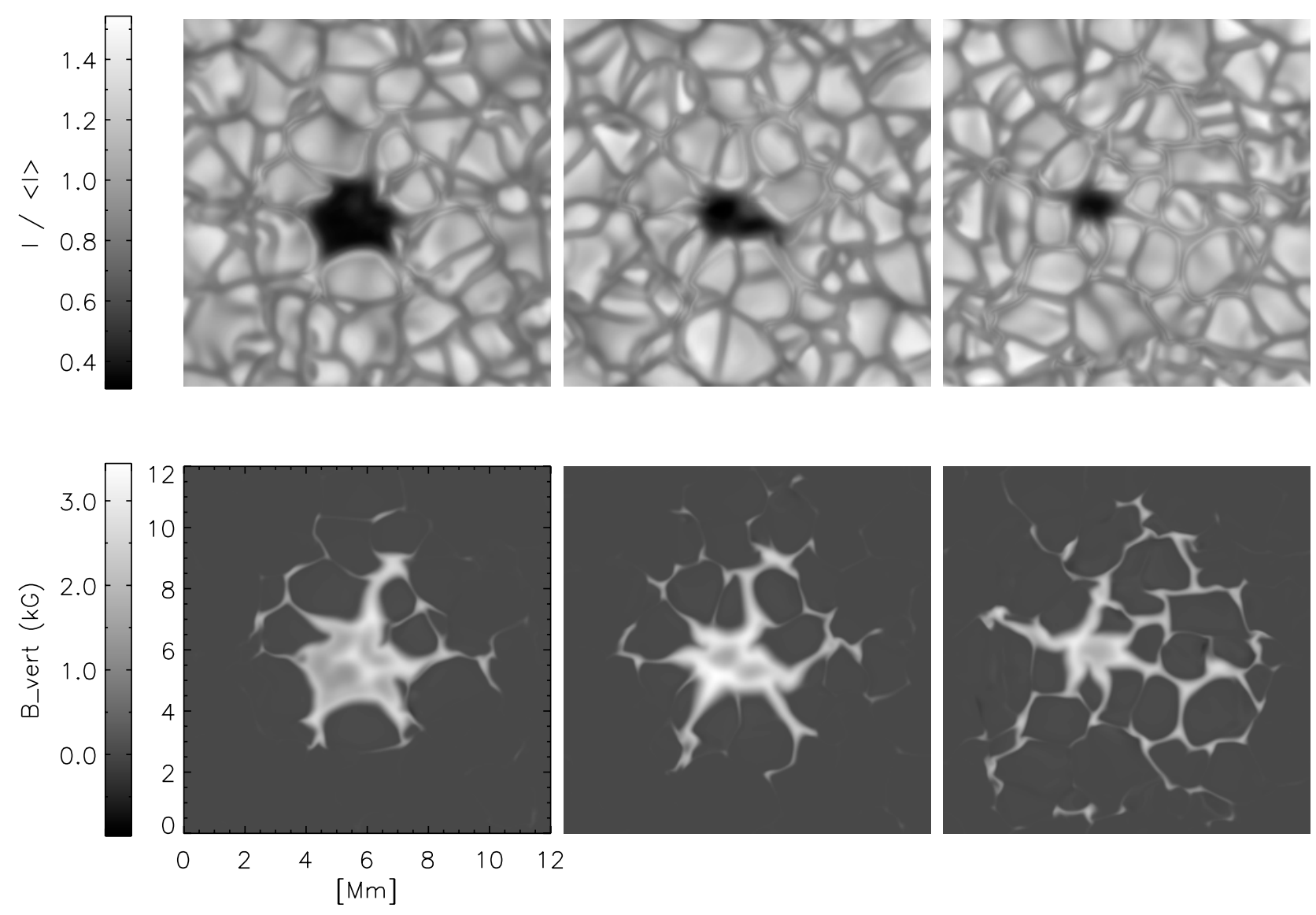

Fig. 1. Time evolution of a simulated pore (run LV1). Shown are maps of the bolometric brightness (wavelength-intergrated intensity in the vertical direction, upper row) and of the vertical component of the magnetic field on the surface defined by the level of Rosseland optical depth unity $\left(\tau_{\mathrm{R}}=1\right.$, lower row). The snapshots are taken $6 \mathrm{~min}$ (left), $27 \mathrm{~min}$ (middle), and $63 \mathrm{~min}$ (right), respectively, after the start of the simulation proper (cf. Sect. 2.3). The pore (as defined by the dark area) has lost almost two thirds of its initial magnetic flux after one hour (see Fig. 2).

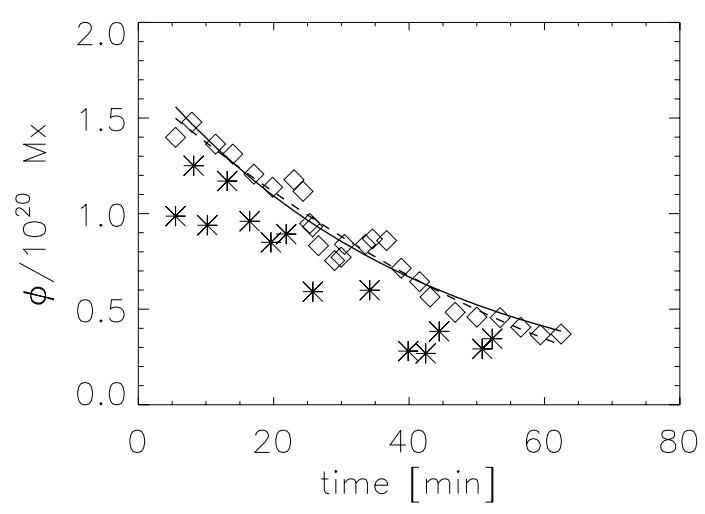

Fig. 2. Magnetic flux of pores (within the region darker than $70 \%$ of the average brightness in the domain) as a function of time for runs LV1 (diamonds) and LP2 (stars).

intensities of our simulated pores are somewhat smaller than results of ground-based observations (e.g., Keil et al. 1999; Keppens \& Martinez Pillet 1996), which could possibly be affected by straylight. When this effect is less important, either because of well-defined straylight correction during a partial eclipse (Bonet et al. 1995), because the less straylight-sensitive infrared range is used (Sobotka et al. 2000), or because the data are taken outside the terrestrial atmosphere (Mathew et al. 2007), the agreement between simulation and observations is better (for structures of similar diameter).

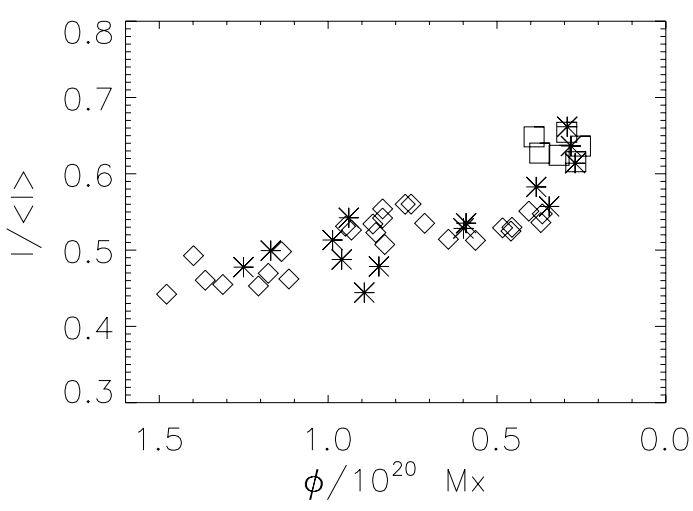

Fig. 3. Mean bolometric brightness as a function of magnetic flux. Points with brightness below $70 \%$ of the overall average brightness of the computational domain have been included in the mean value over the pore. Shown are results from runs LV1 (diamonds), LP2 (stars), and SV2 (squares). On the abscissa, the magnetic flux decreases to the right to be consistent with the direction of the time evolution shown in Fig. 2.

\subsection{Magnetic field, velocity and brightness structure}

We continue with a more detailed discussion of the physical properties of the pore simulated in run LV1, considering a snapshot taken $27 \mathrm{~min}$ after the start of the simulation proper (corresponding to the middle panel of Fig. 1). 

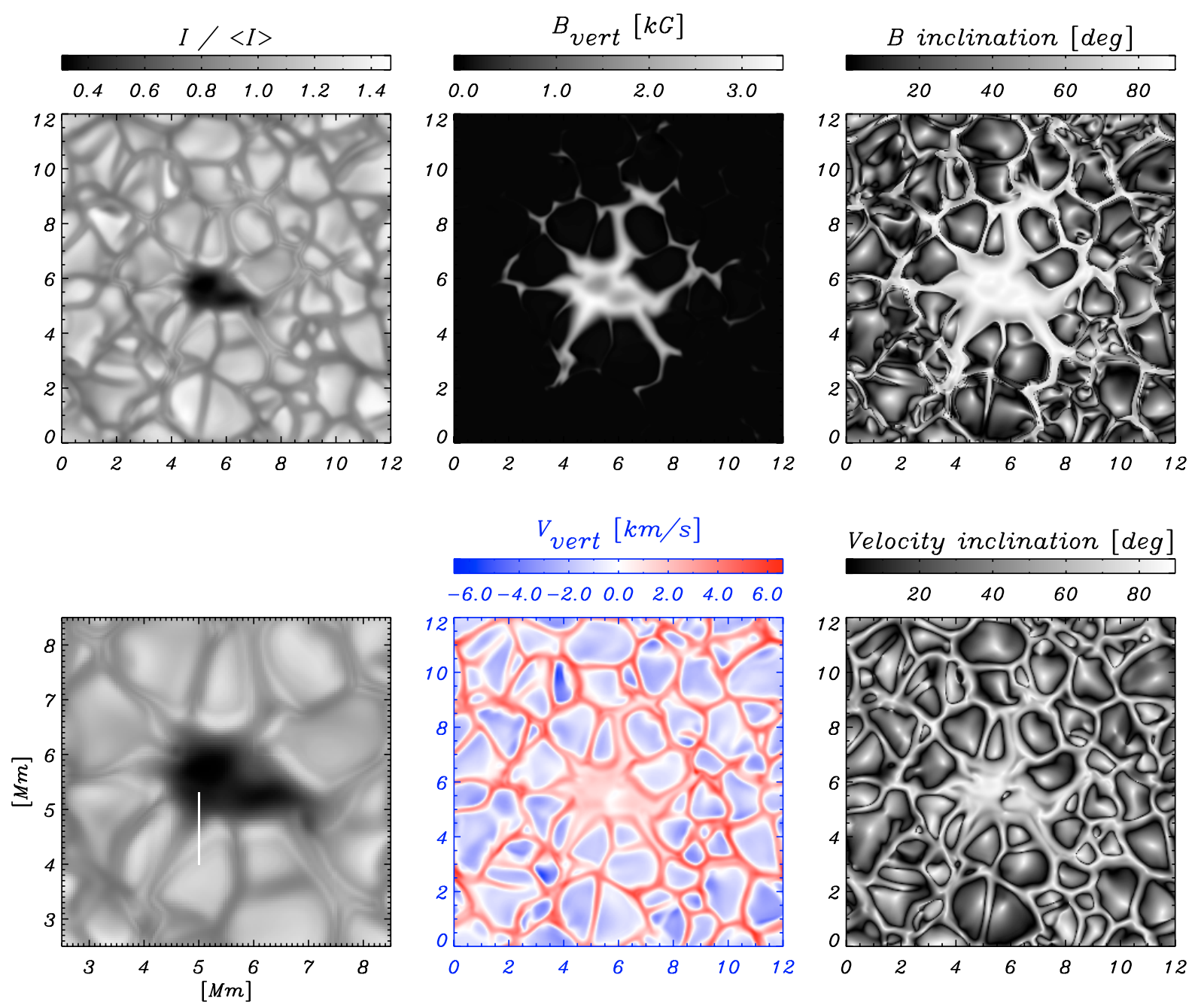

Fig. 4. Maps of various quantities at the visible surface (run LV1, middle snapshot in Fig. 1) upper left: bolometric brightness; upper middle: vertical component of the magnetic field; upper right: inclination angle of the magnetic vector with respect to the horizontal plane; lower left: zoom of the brightness image, indicating the location of the vertical cut shown in Fig. 5; lower middle: vertical component of the flow velocity (red: downflow, blue: upflow); lower right: inclination angle of the velocity vector with respect to the horizontal plane. All quantities (except brightness) are shown on the surface corresponding to $\tau_{\mathrm{R}}=1$.

Table 2. Average relative continuum intensities, $\left\langle I_{\mathrm{c}, \text { pore }}\right\rangle /\left\langle I_{\mathrm{c}}\right\rangle$ for the three snapshots from run LV1 shown in Fig. 1. The numbers in parantheses give the effective diameter of the dark region (diameter of a circle enclosing the same area, in arcsec) with an intensity less than $70 \%$ of the mean intensity at $540 \mathrm{~nm}$.

\begin{tabular}{c|ccc}
\hline \hline$\lambda[\mathrm{nm}]$ & $\# 1\left(3.8^{\prime \prime}\right)$ & $\# 2\left(3^{\prime \prime}\right)$ & $\# 3\left(2.1^{\prime \prime}\right)$ \\
\hline 540 & 0.35 & 0.41 & 0.45 \\
600 & 0.39 & 0.46 & 0.49 \\
630 & 0.40 & 0.48 & 0.51 \\
670 & 0.43 & 0.50 & 0.53 \\
800 & 0.49 & 0.56 & 0.59 \\
1550 & 0.71 & 0.73 & 0.76 \\
\hline
\end{tabular}

Figure 4 shows maps of various physical quantities on the surface of Rosseland optical depth equal to unity, $\tau_{\mathrm{R}}=1$. The pore is in the process of losing magnetic flux into the adjacent intergranular lanes, leading to a starfish-like appearance in the magnetic field map. There is an overall downflow of the order of $1 \mathrm{~km} \mathrm{~s}^{-1}$ in the pore, which indicates that it has not yet reached a stationary structure: while the magnetostatic force balance is readily achieved, the proper mode of magneto-convective energy transport and the corresponding stationary thermal structure is only established after about a Kelvin-Helmholtz time of the order of $10 \mathrm{~h}$. Local variations of the downflow speed affect the magnetic field in the visible layers, leading to inhomogeneities of the magnetic field strength of the order of $\pm 20 \%$ in the dark part of the pore.

The field is largely vertical where it is strong: in the pore, in its "tentacles" connecting to intergranular lanes, and in the surrounding network of downflows. Around the pore, we have thin downflow lanes, immediately adjacent to normal granules. Very similar properties have been found observationally by Sankarasubramanian \& Rimmele (2003).

A more detailed view is provided by a two-dimensional vertical cut through the pore along the white line drawn in the lower left panel of Fig. 4. Various quantities on this cut are shown in the left and middle panels of Fig. 5. The horizontal profile of the bolometric intensity given on the lower left panel shows a decrease to about $40 \%$ of the quiet-Sun level in the interior of the pore. Near its boundary (around $x \simeq 4.35 \mathrm{Mm}$ ), the edge brightening of the adjacent granule with a contrast of about $5 \%$ is apparent (cf. the brightness maps in Figs. 1 and 4). Comparison of the isotherm for $T=7400 \mathrm{~K}$ (white curve) and the contour 


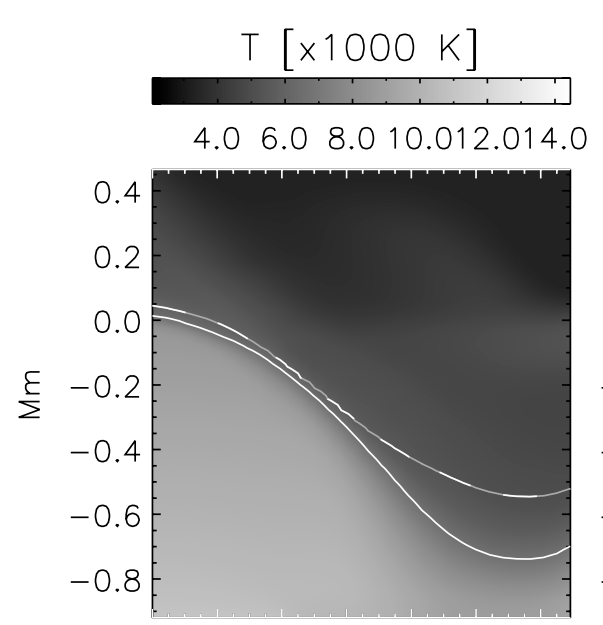

$4.0 \quad 4.2 \quad 4.4 \quad 4.6 \quad 4.85 .05 .2$ $\operatorname{Min}$

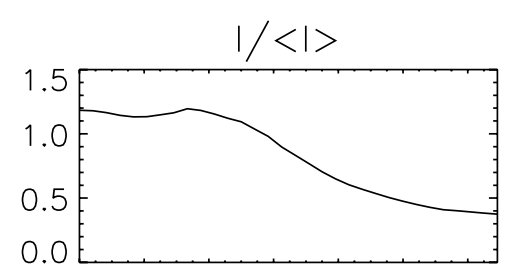

$\begin{array}{lllllll}4.0 & 4.2 & 4.4 & 4.6 & 4.8 & 5.0 & 5.2\end{array}$ $\mathrm{Mm}$
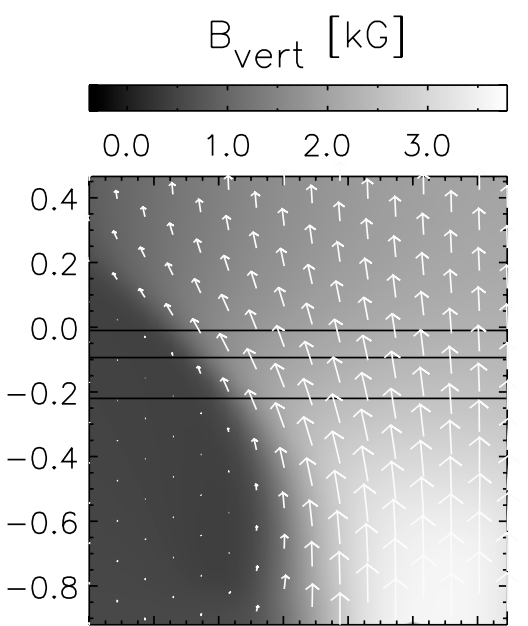

$\begin{array}{lllllll}4.0 & 4.2 & 4.4 & 4.6 & 4.8 & 5.0 & 5.2\end{array}$ $\mathrm{Mm}$
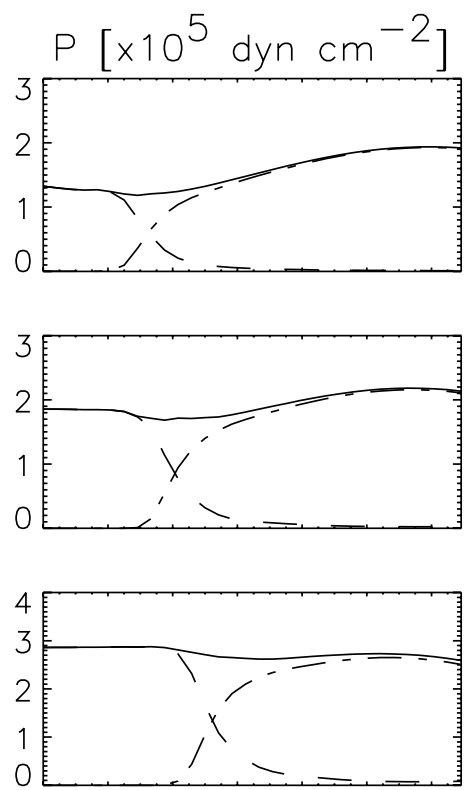

$4.04 .2 \quad 4.4 \quad 4.6 \quad 4.85 .05 .2$
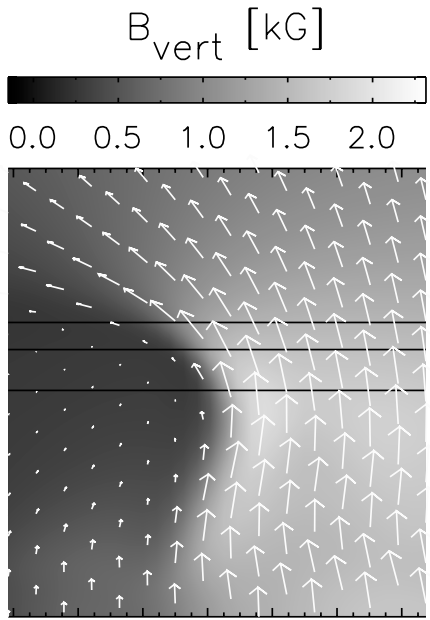

$\begin{array}{lllllll}3.6 & 3.8 & 4.0 & 4.2 & 4.4 & 4.6 & 4.8\end{array}$ $\mathrm{Mm}$
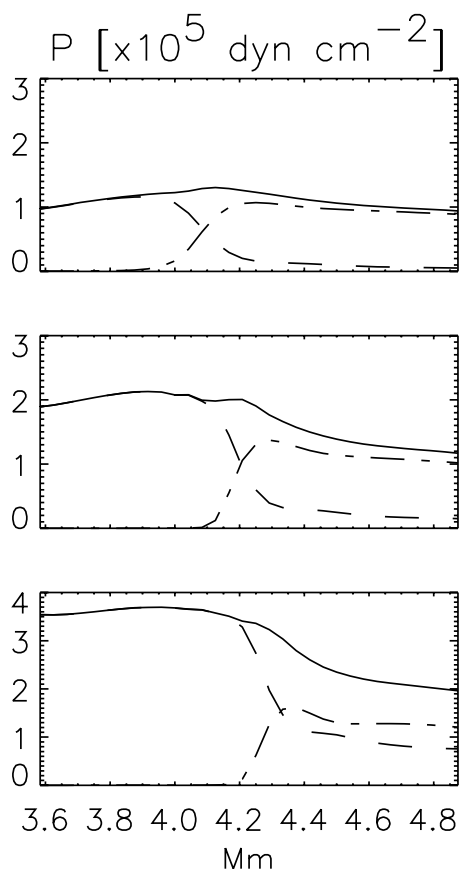

Fig. 5. Vertical cuts through simulated pores. Left panels: grey-scale plot of temperature for a cut along the white line in the lower left panel of Fig. 4 (run LV1). The white-grey dashed line shows the level $\tau_{\mathrm{R}}=1$ and the solid white line represents the isotherm for $T=7400 \mathrm{~K}$. The plot below gives the normalized horizontal brightness profile. Middle panels: grey-scale plot of the vertical magnetic field strength with field vectors projected onto the vertical plane. The plots below show the horizontal profiles of gas pressure (dashed), magnetic pressure (dash-dotted), and total pressure (full) at three geometrical heights, indicated by the horizontal lines in the upper part. Right panels: same for a snapshot from run LP2. The zero point $(z=0)$ of the height scale roughly corresponds to the average quiet-Sun level of $\tau_{\mathrm{R}}=1$.

line for $\tau_{\mathrm{R}}=1$ (white-grey dashed curve) in the upper left panel shows that the edge brightening is due to the different slope of the respective contour lines near the rim of the pore: owing to the larger transparency of the diluted and cool plasma in the overlying magnetic structure, the $\tau_{\mathrm{R}}=1$ contour descends somewhat more steeply into the pore interior than the local isotherms and therefore reaches a somewhat higher temperature in the region of the bright rim. In addition, there is radiative heating of the plasma in the pore near its boundary. This situation is analogous to that found by Deinzer et al. (1984); Knölker \& Schüssler (1988) in the case of flux sheet models. Further inward into the pore, the situation is reversed: here, the isotherms descend more sharply than the iso- $\tau$ lines, leading to decreasing temperature at the $\tau_{\mathrm{R}}=1$ surface and thus lower brightness. A discussion of bright rings in sunspot models along these lines has also been given by Pizzo (1986, see his Fig. 10).

The map of the magnetic field given in top panel of the middle column of Fig. 5 indicates a strong expansion of the magnetic structure above $z \simeq-400 \mathrm{~km}$, the approximate height of the level $\tau_{\mathrm{R}}=1$ in the pore's interior. Horizontal profiles of gas, magnetic and total pressure, respectively, at the heights indicated by the horizontal lines are shown in the lower part of the middle column. The lower two cuts are roughly in accordance with total pressure balance between the pore and its surroundings, i.e., the absence of significant magnetic tension effects. The cut at $z=0$ already shows the effect of the vertical-field upper boundary condition in a nearly force-free configuration with low plasma beta: as the field lines start to bend inward in order to become vertical 


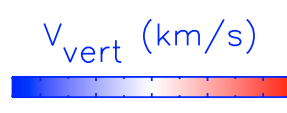

$\begin{array}{lllll}-4 & -2 & 0 & 2 & 4\end{array}$
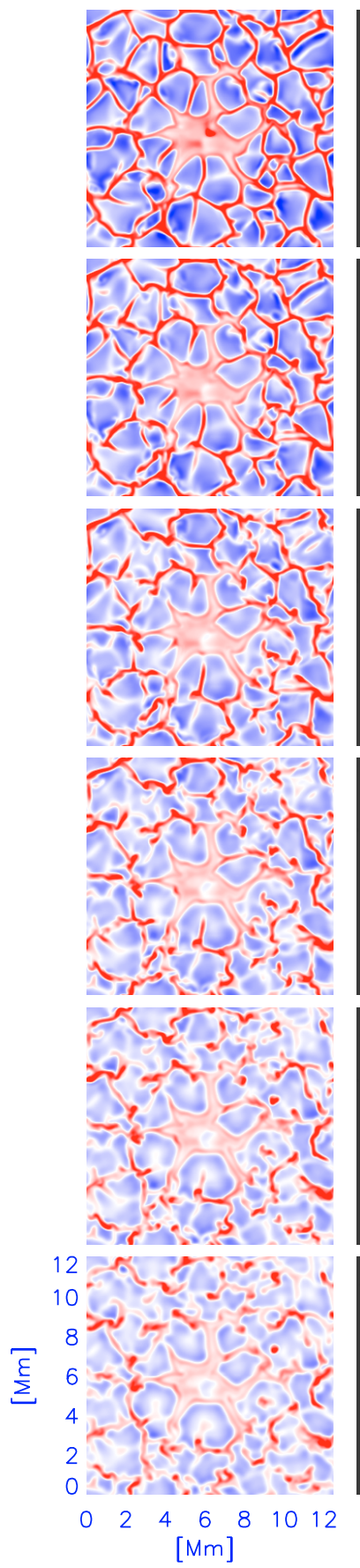

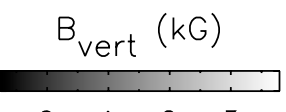

$\begin{array}{llll}0 & 1 & 2 & 3\end{array}$
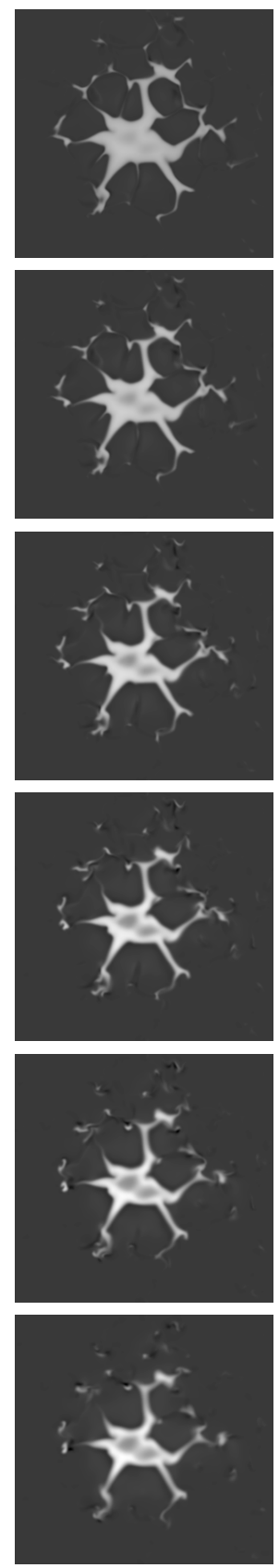
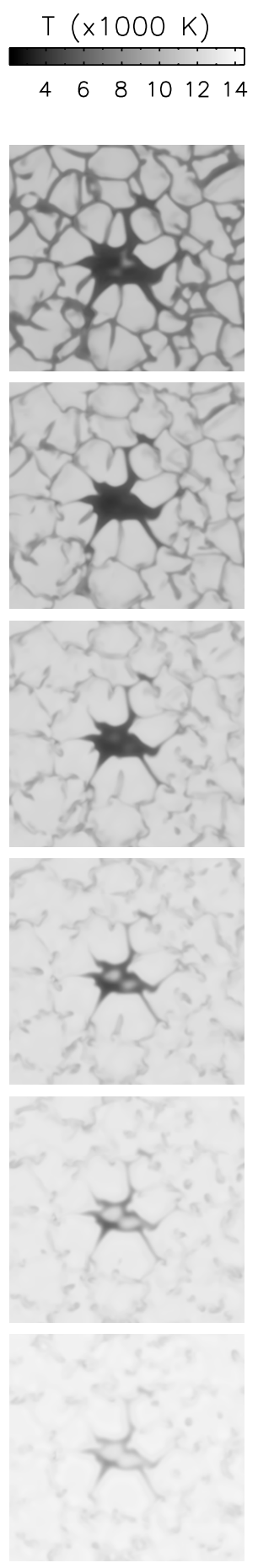

$\mathrm{d}=-120 \mathrm{~km}$

$\mathrm{d}=0 \mathrm{~km}$

$\mathrm{d}=120 \mathrm{~km}$

$\mathrm{d}=240 \mathrm{~km}$

$\mathrm{d}=360 \mathrm{~km}$

$\mathrm{d}=480 \mathrm{~km}$

Fig. 6. Depth structure of the simulated pore (run LV1, middle snapshot in Fig. 1), illustrated by horizontal cuts at various geometrical depths. Shown are maps of vertical velocity (left column), vertical magnetic field (middle column), and temperature (right column). Subsequent cuts are seperated by $120 \mathrm{~km}$ in depth. The second row from the top (at depth $d=0$ ) roughly corresponds to the visible surface, $\tau_{\mathrm{R}}=1$, outside the pore. at the top, the resulting inward curvature force has to be balanced by an outward decrease of the magnetic pressure.

The situation is somewhat different for a simulation with a potential field at the upper boundary. The panels in the righthand column of Fig. 5 show a vertical cut through a snapshot from run LP2. The expansion of the field with height is no longer constrained by the upper boundary condition as in the case of run LV1, so that the curvature force is directed outward in the upper part of the pore. It is balanced by an inward decrease of the total pressure. The slight constriction of the pore around the height level of $\tau_{\mathrm{R}}=1$ within the pore is reminiscent to a similar property of the magnetostatic models of Pizzo (1986). In our case, however, it is presumably caused by a somewhat larger local external pressure (possibly due to a large granule) in this height range. Anyway, these differences in the magnetic structure are sufficiently small so that they do not significantly affect the observable properties of the pore.

Figure 6 illustrates the structure of the pore (run LV1) at various depths, ranging from about $100 \mathrm{~km}$ above the undisturbed solar surface (top row) to about $500 \mathrm{~km}$ below. Over this range of heights, the magnetic structure remains very similar. Hotter plasma has risen in two plume-like structures in the lower layers 

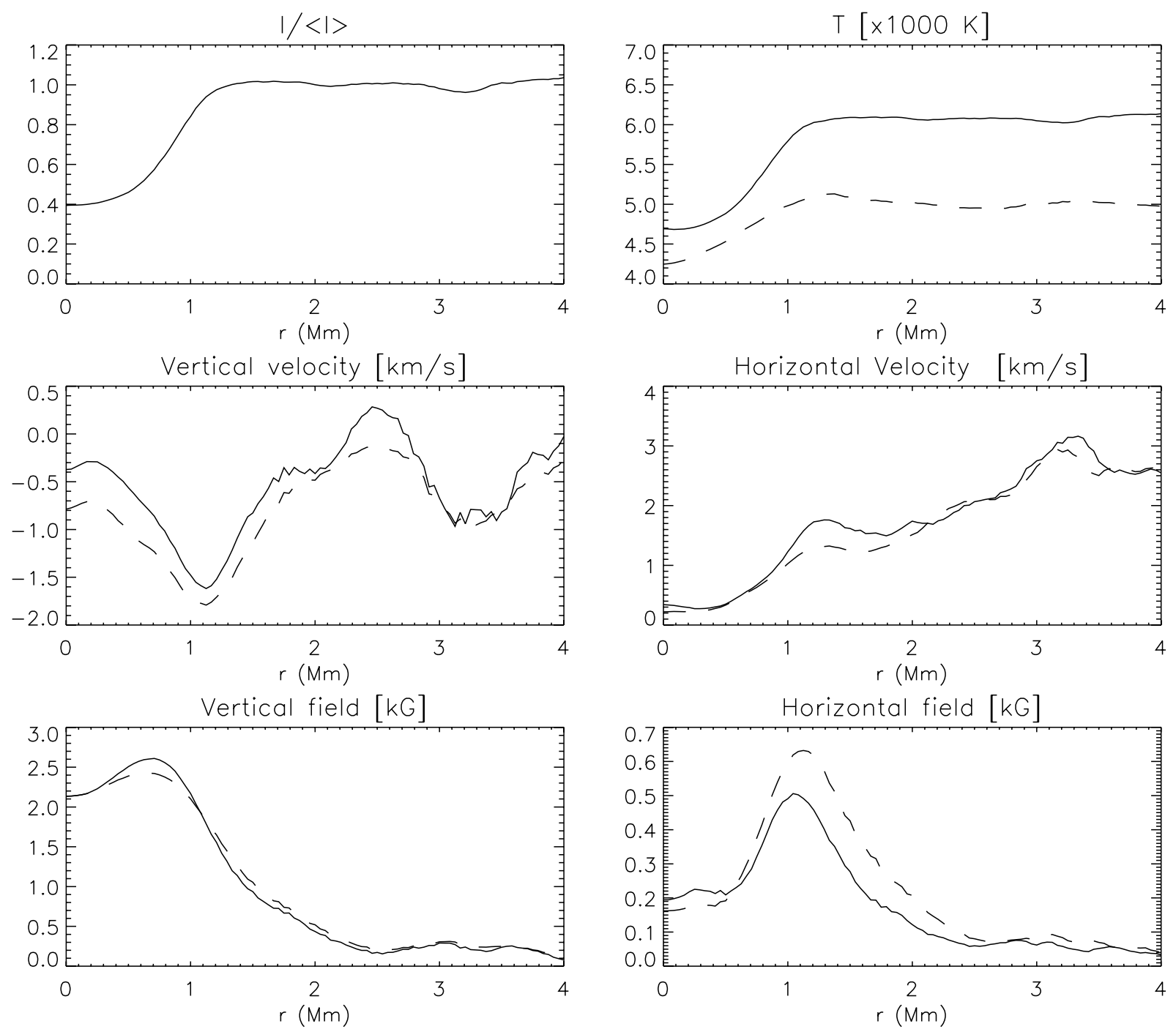

Field inclination $[\mathrm{deg}]$
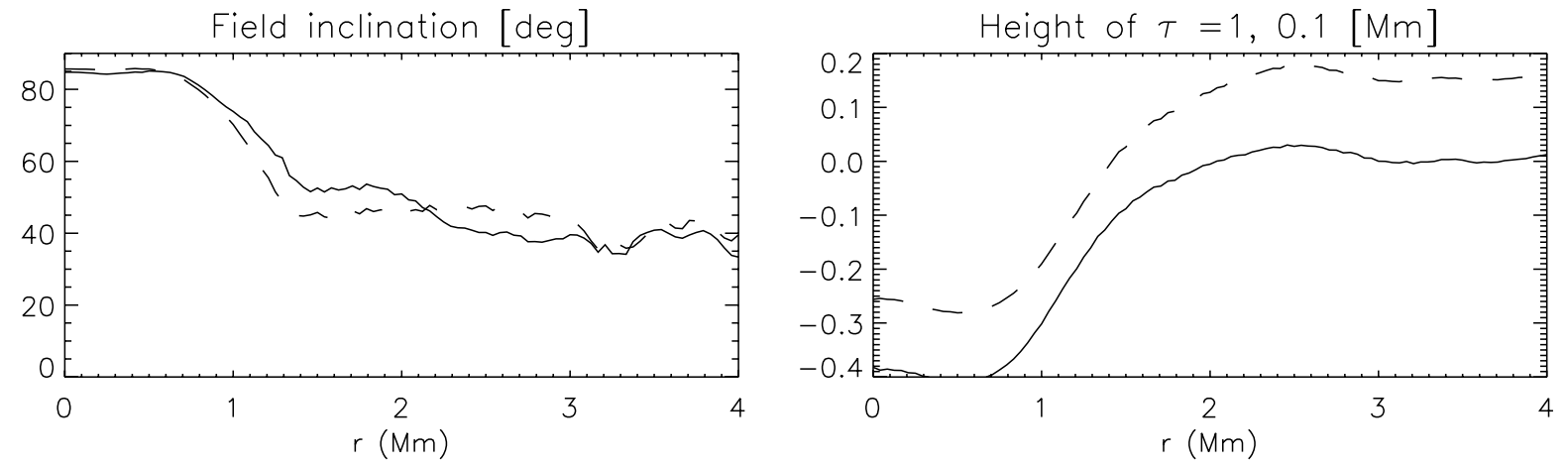

Fig. 7. Azimuthally averaged profiles radially outward from the center of the pore (run LV1, middle snapshot in Fig. 1). Top left: vertically emerging bolometric intensity, normalized to the corresponding average quiet-sun intensity. The quantities given in the other plots are identified at the top of the corresponding panels. Solid lines represent profiles at the level $\tau_{\mathrm{R}}=1$, dashed lines correspond to $\tau_{\mathrm{R}}=0.1$. Inclinations are given with respect to the horizontal plane. In this figure, positive vertical velocities correspond to upflows and positive horizontal velocities to flows directed toward the center of the pore.

of the pore interior, its expansion leading to a local weakening of the field (cf. Schüssler \& Vögler 2006).

We have determined mean horizontal profiles of various quantities within the pore by performing radial cuts through the geometrical center of the pore (i.e., the center of gravity of the region with an intensity below $70 \%$ of the mean) and averaging over azimuth. The results are shown in Fig. 7. The vertical bolometric intensity given in the upper left panel falls to about $40 \%$ of the average quiet-Sun value at the center of the pore. The other panels in Fig. 7 show the radial profiles of azimuthally averaged quantities at the surfaces of Rosseland optical depth unity $\left(\tau_{\mathrm{R}}=1\right.$, full lines) and $\tau_{\mathrm{R}}=0.1$ (dashed lines), respectively. Towards the pore center, the temperature (at constant optical depth) drops by $\sim 1300 \mathrm{~K}\left(\right.$ at $\left.\tau_{\mathrm{R}}=1\right)$ and $\sim 700 \mathrm{~K}$ (at $\tau_{\mathrm{R}}=0.1$ ), respectively, indicating a flatter temperature gradient in the pore in comparison to its environment (cf. Fig. 9). 

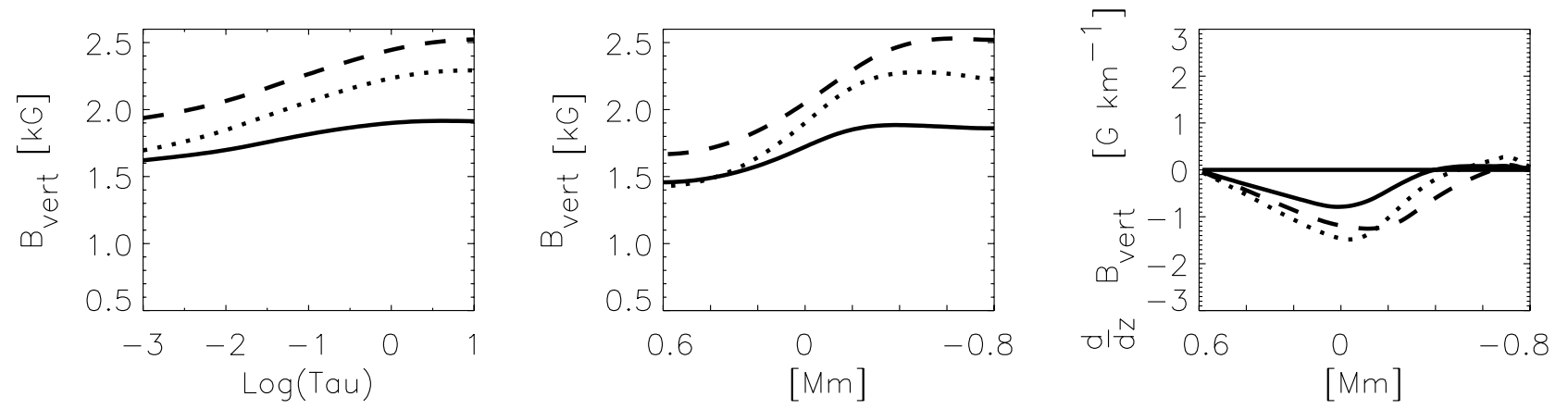

Fig. 8. Height-dependence of the horizontally averaged vertical magnetic field component (run LV1). Averaging is carried out over the area with brightness less than $70 \%$ of the mean. Shown is the vertical field component as a function of Rosseland optical depth (left panel) and as a function of geometrical height (middle panel). The height gradient is given on the right panel. The three curves represent the snapshots shown in Fig. 1, which correspond to $6 \mathrm{~min}$ (solid curve), $27 \mathrm{~min}$ (dashed) and $63 \mathrm{~min}$ (dotted), respectively, after the start of the simulation proper.

The contours of constant optical depth correspond to a Wilson depression of about $400 \mathrm{~km}$.

The velocity structure is characterized by a downflows around the pore, which are supplied by a radial inflow. This kind of flow pattern has also been found in observations (Sobotka et al. 1999; Dorotovič et al. 2002; Sankarasubramanian \& Rimmele 2003; Hirzberger 2003). Within the pore, the flow is mostly vertical and directed downward. Systematic downflows of a few $100 \mathrm{~m} \mathrm{~s}^{-1}$ within pores have been reported by some authors (e.g., Keil et al. 1999), while other observers do not find significant flows (e.g., Hirzberger 2003).

The mean vertical magnetic field ranges from about $2100 \mathrm{G}$ to $2600 \mathrm{G}$ in the interior of the pore. The apparently smooth outward decrease of the field (and of other quantities) for $r \gtrsim 1 \mathrm{Mm}$ is an artifact of averaging over the elongated and starfish-like magnetic shape of the pore. The field lines become less inclined toward the edge of the pore; however, the inclination angle (with respect to the horizontal plane) does not fall significantly below $\simeq 50 \mathrm{deg}$ at the rim of the pore, so that no sign for the formation of horizontal, penumbra-like fields can be detected. In particular, we do not find values down to $30 \mathrm{deg}$ as suggested by Sütterlin (1998). On the other hand, the profiles of magnetic field and inclination for our simulated pore are roughly consistent with the average profiles shown by Keppens \& Martinez Pillet (1996, their Fig. 3) based on observations of 51 pores.

All these results are fairly independent of the choice of the upper boundary condition: snapshots from the runs with the potential-field condition show qualitatively similar profiles as those in Fig. 7.

In order to determine the mean depth-profiles of the vertical magnetic field, we have calculated horizontal averages over the area with (bolometric) intensity less than $70 \%$ of the mean. The results are shown in Fig. 8 as functions of Rosseland optical depth and geometrical depth, respectively, together with the depth gradient. The three curves correspond to the three snapshots shown in Fig. 1. They indicate a considerable temporal variation of the field strength at a given height: an initial increase of the field strength, due to the net downflow driven by surface cooling (thermally non-stationary situation), is followed by a later decline as the pore shrinks by shedding magnetic flux to the surrounding intergranular downflow network. The range of field strengths in the observable layers, roughly $1700-2300 \mathrm{G}$, is consistent with the values reported by observers (e.g., Brants \& Zwaan 1982; Muglach et al. 1994; Keppens \& Martinez Pillet 1996; Sütterlin 1998). The field strength decreases with geometrical height most rapidly near the visible solar surface, where the external pressure scale height drops steeply. However, the maximum height gradient of $1-1.5 \mathrm{G} \mathrm{km}^{-1}$ in the simulated pores is smaller (by a factor of 2-3 than the value derived by Sütterlin (1998) using an inversion of observed Stokes profiles. This result is not an artifact of the vertical-field upper boundary condition of run LV1, since the runs with the potential-field boundary condition show almost the same height gradient in the observable layers. On the other hand, values of the height gradient similar to our results are also exhibited by the magnetostatic equilibrium models of Pizzo (1986).

The thick lines in Fig. 9 show, for the same snapshots as in the previous figure, optical depth profiles of horizontally averaged density, temperature and pressure, respectively, in the pore, determined analoguously to the magnetic field profiles discussed above. The thin solid lines represent the empirical models of Sütterlin (1998), based on observational data for three pores, and the thin dashed curves represent the respective average profiles from the simulation outside the pore. The pressure and density profiles match the empirical models quite well. The empirical temperatures (middle panel) depend on the size of the observed pore. In the deep layers, the simulation is in good agreement with the model for the smallest pore in the sample of Sütterlin (1998) with a diameter of about $2^{\prime \prime}(\sim 1500 \mathrm{~km})$. In the photosphere, the temperatures of the simulated pore range between this model and the models for the two somewhat larger pores ( $3^{\prime \prime}$ and $4.5^{\prime \prime}$, respectively). However, since we have considered grey radiative transfer and thus ignore backwarming effects, the temperature in the simulated pore is probably about $200 \mathrm{~K}$ too low in the deeper photosphere (cf. Vögler 2004a). The temperature rise around $\log \tau_{\mathrm{R}}=-2$ is an artifact of the minimum-density condition introduced to avoid exceedingly small time steps: local downflows lead to adiabatic heating. This effect is much smaller in non-grey simulations (cf. Cameron et al. 2007).

\subsection{Center-to-limb variation of the continuum intensity}

Figure 10 shows a snapshot from the simulation run LP2 in the continuum intensity at $500 \mathrm{~nm}$ for three inclination angles ( $\mu=\cos \theta=0.7,0.5,0.3$, respectively) of the line of sight. One can clearly recognize the "facular" brightening of the limbward granules adjacent to the pore, which is due to the enhanced transparency of the tenuous pore plasma, so that the hot granular plasma behind the pore leads to higher brightness (Spruit 1976; Keller et al. 2004; Carlsson et al. 2004; Steiner 2005). When normalized with the average intensity at the same limb distance (right panel of Fig. 10), the contrast of these "bright 

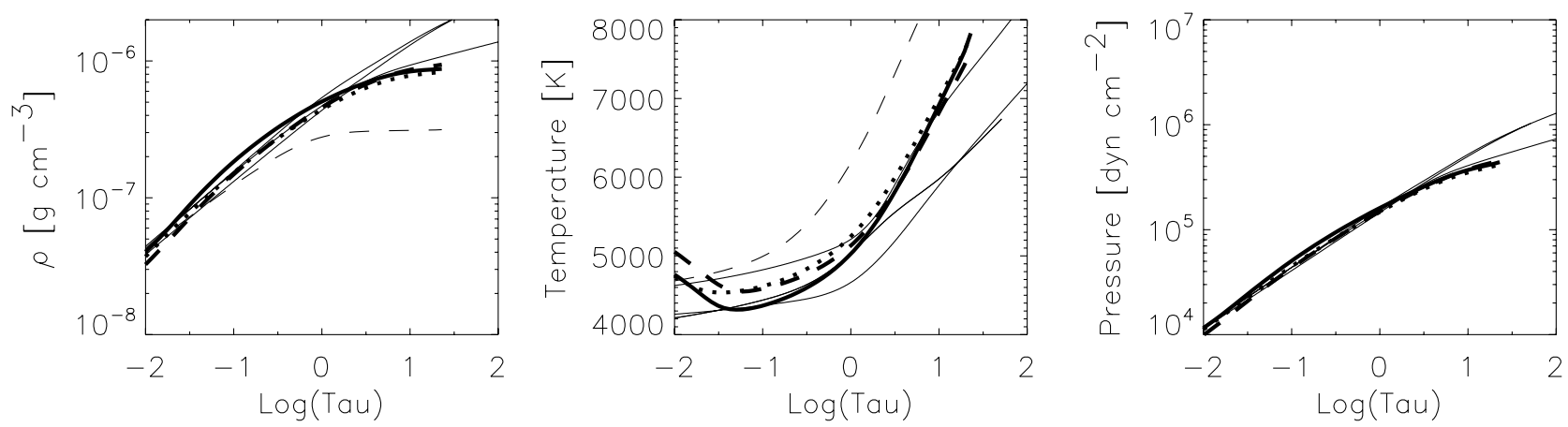

Fig. 9. Comparison between a simulated pore (run LV1) and empirical models. The thick (solid, dashed, and dotted) lines give the horizontally averaged density (left), temperature (middle), and pressure (right) as functions of Rosseland optical depth for the same snapshots as in the previous figure. The thin dashed line shows the corresponding average profiles in the simulation domain outside the pore. The thin solid lines show empirical models derived by Sütterlin (1998) from observations of three pores of different size.
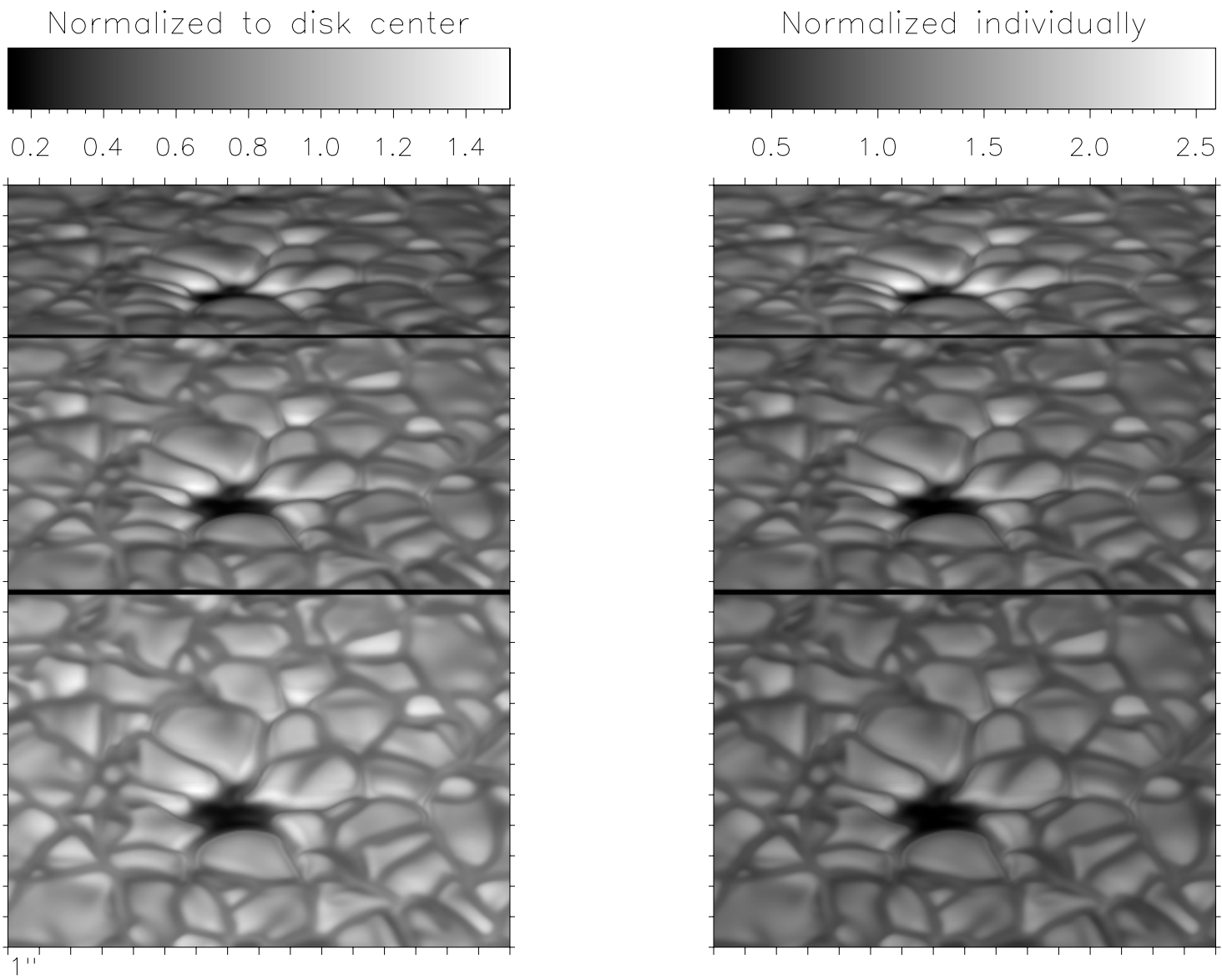

Fig. 10. Center-to-limb variation of a simulated pore (snapshot from run LP2). Shown are continuum intensity images at $500 \mathrm{~nm}$ for $\mu=\cos \theta=0.7$ (bottom), 0.5 (middle), and 0.2 (top). On the left panel, the intensities are normalized with respect to the average disk center intensity, while the right panel gives intensities normalized to the averaged intensities at the same inclination (limb distance).

walls" strongly increases towards the limb. The increase of contrast is also apparent from Fig. 11, which shows intensity profiles for three limb distances along the same cut through the pore and the adjacent granule pattern. The intensity is high on the centerward parts of the granules behind the pore and gradually decreases in the limbward direction.

\section{Discussion and conclusions}

Our simulations with a radiative MHD code are the first models of solar pores including most of the relevant physics (3D, compressibility, radiation, partial ionization, surrounding convection). Therefore, it is of interest to compare the results with previous simplified models.

The overall magnetic structure of our simulated pores resembles the axisymmetric models of Simon et al. (1983): a largely force-free (potential) field in the photospheric layers of the pore interior and a net magnetic force acting near the rather sharp transition between the flux concentration from the exterior. When averaging over the internal inhomogeneities of the field strength due to the local flows, we find qualitatively similar radial profiles as a function of radius: the vertical field 

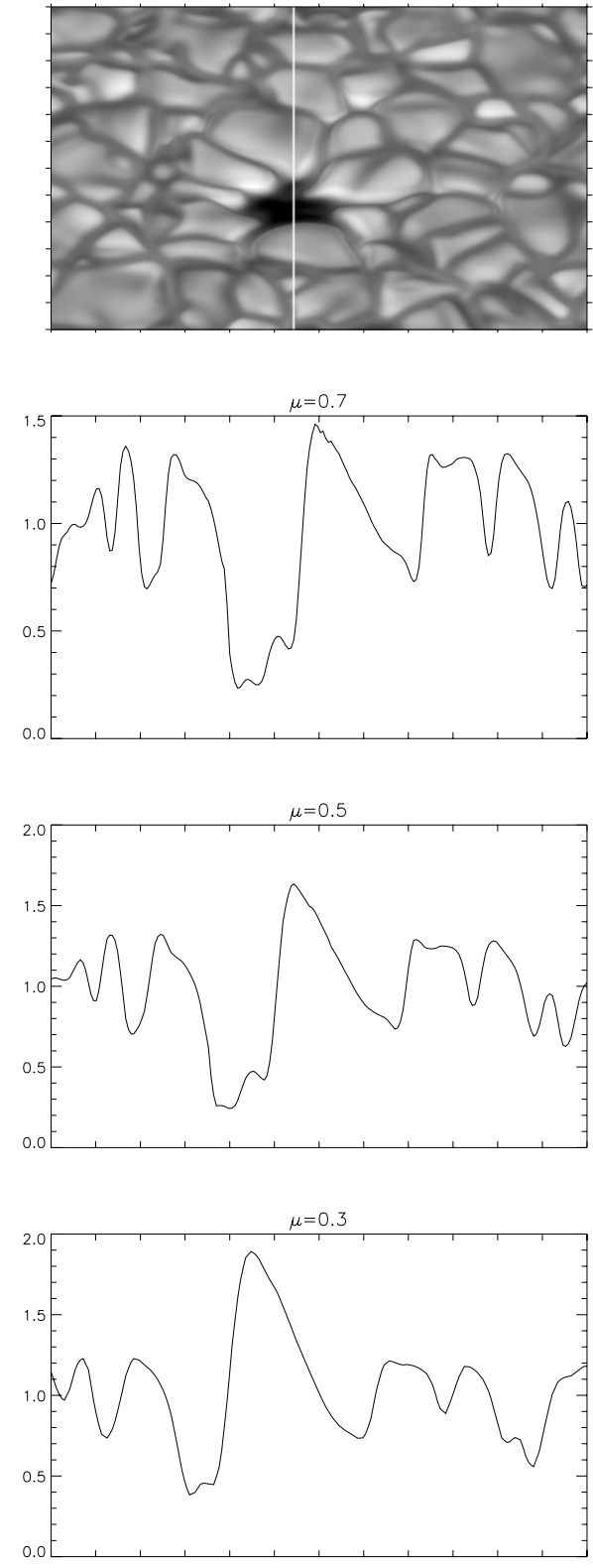

Fig. 11. Continuum intensity at $500 \mathrm{~nm}$ along a cut through the simulated pore (snapshot from run LP2), for three values of $\mu=\cos \theta$. The cut is indicated for $\mu=0.7$ in the top panel. The profiles are individually normalized by the mean intensity at the same limb distance. The geometric foreshortening is compensated in the three profiles.

component remains roughly constant over the pore interior and drops sharply over a few $100 \mathrm{~km}$ horizontal distance towards the exterior, while the radial component shows a maximum near the tube boundary (compare our Figs. 5 and 7 with Figs. 2c and 4c in Simon et al. 1983).

Pizzo (1986) determined magnetostatic equilibria of axisymmetric flux tubes using empirical models for the internal and external temperature, which are vertically shifted with respect to each other by a fixed amount (roughly corresponding to the Wilson depression). His pore-sized models exhibit a distinct hourglass shape: the assumption of a large gas pressure deficit around optical depth unity in the flux tube leads to a strong local constriction of the flux tube, so that the inward directed pressure force is balanced by the magnetic curvature force. In most of our thermodynamically consistent models, we find significantly smaller pressure deficits, so that no local constrictions appear. An exception is the simulation with potential-field upper boundary condition, which expands more rapidly with height and shows a slight constriction near optical depth unity within the pore.

The height gradient of the field strength in the upper (expanding) part of Pizzo's models is consistent with the values shown by our simulated pores (of the order of $1 \mathrm{G} \mathrm{km}^{-1}$ ). The much stronger decline of the field suggested by Sütterlin (1998) from inversions based upon observational data is difficult to reconcile with these results. On the other hand, for the photospheric layers of sunspot umbrae, values in the range $1-3 \mathrm{G} \mathrm{km}^{-1}$ have been derived by various observers (cf., Solanki 2003); these are more consistent with our results and with those of Pizzo (1986).

Even though they include much of the relevant physics, our simulations of solar pores are still of an exploratory nature and limited in various respects. The main limitation is the grid resolution with a horizontal spacing of about $42 \mathrm{~km}$ for the runs discussed here in detail. Such a coarse resolution (entailing a correspondingly rather high magnetic difusivity parameter) prevents the formation of narrow upflow plumes that would appear as bright patches similar to umbral dots of sunspots (Schüssler \& Vögler 2006). This could also possibly suppress the appearance of penumbra-like structures for the larger simulated pores, if the penumbra formation depends on the onset of particular modes of magneto-convection involving small spatial scales (e.g., Rucklidge et al. 1995).

Another limitation concerns the effect of the initial state on the structure of the deeper layers and the energy transport within the pore: the thermal relaxation times are of the order of hours, so that no full relaxation occurs during the lifetime of the structure. Although we have evolved the system in 2D for 10-15 h before starting the $3 \mathrm{D}$ simulations, it cannot be excluded that the results are still affected by our arbitrary choice of the initial entropy distribution in the pore. This would be most relevant if the true mode of magneto-convective energy transport could not fully develop in the simulation because of too coarse grid resolution and/or the limitations of 2D dimensionality during the initial relaxation phase.

The lower boundary of the computational box is another limiting factor: further simulation runs will have to be carried out in order to determine the effect of the chosen depth for this boundary and the dependence on the specific choice of the (reduced) energy transport into the pore from below.

Finally, we summarize the main results of this work as follows:

- The simulated pores appear as dark structures surrounded by edge-brightened granules. Their intensity contrasts in the visible and infrared spectral ranges are consistent with (straylight-corrected) observational results. Larger pores are darker than smaller ones. When observed near the solar limb, the pores show a strong "facular" brightening of the granules behind them.

- The average magnetic field strength in the lower photosphere is in the range $2000 \pm 300 \mathrm{G}$. The field is predominantly vertical, the horizontal component reaching a maximum near the periphery of the pore. The field inclination (with respect to the horizontal) remains larger than $\simeq 50 \mathrm{deg}$; there is no indication for the formation of a penumbra-like structure.

- The average optical depth profiles of temperature, pressure, and density are consistent with semi-empirical models based upon spectroscopic observations. Deviations of a few $100 \mathrm{~K}$ are found for the temperature in the low photosphere. 
- The simulated pores are surrounded by downflows at their periphery. These are maintained by horizontal flows towards the pore, which, at the same time, contribute to keeping the magnetic structure together. Within pores, we find moderate downflows of the order of up to about $1 \mathrm{~km} \mathrm{~s}^{-1}$, indicating a slow approach to a thermally relaxed configuration.

- The simulated pores decay be losing flux from the periphery of the magnetic structure into adjacent intergranular lanes, akin to a "turbulent erosion" process.

Acknowledgements. P. Sütterlin kindly made available to us his pore models in electronic form, which were used in Fig. 9.

\section{References}

Beckers, J. M., \& Schröter, E. H. 1968, Sol. Phys., 4, 142

Bercik, D. J. 2002, Ph.D. Thesis, Michigan State University

Bercik, D. J., Nordlund, A., \& Stein, R. F. 2003, in Local and Global Helioseismology: the Present and Future, ed. H. Sawaya-Lacoste (European Space Agency, ESA SP-517), 201

Bonet, J. A., Sobotka, M., \& Vazquez, M. 1995, A\&A, 296, 241

Brants, J. J., \& Zwaan, C. 1982, Sol. Phys., 80, 251

Bray, R. J., \& Loughhead, R. E. 1964, Sunspots, The International Astrophysics Series (London: Chapman \& Hall)

Cameron, R., Vögler, A., Shelyag, S., \& Schüssler, M. 2004, in The Solar-B Mission and the Forefront of Solar Physics, ed. T. Sakurai (San Francisco: ASP), ASP Conf. Ser., 57

Cameron, R., Vögler, A., \& Schüssler, M. 2007, in Convection in Astrophysics, ed. F. Kupka, I. Roxburgh, \& K. Chan (Cambridge/UK: Cambridge Univ. Press), IAU Symp., 239, 475

Carlsson, M., Stein, R. F., Nordlund, Å., \& Scharmer, G. B. 2004, ApJ, 610, L137

Caunt, S. E., \& Korpi, M. J. 2001, A\&A, 369, 706

Cheung, A. 2006, Ph.D. Thesis, University of Göttingen, Germany, http://webdoc.sub.gwdg.de/diss/2006/cheung

Cheung, M. C. M., Schüssler, M., \& Moreno-Insertis, F. 2007a, A\&A, 467, 703

Cheung, M. C. M., Schüssler, M., \& Moreno-Insertis, F. 2007b, A\&A, 461, 1163

Deinzer, W., Hensler, G., Schüssler, M., \& Weisshaar, E. 1984, A\&A, 139, 426

Dorotovič, I., Sobotka, M., Brandt, P. N., \& Simon, G. W. 2002, A\&A, 387, 665

Hirzberger, J. 2003, A\&A, 405, 331

Keil, S. L., Balasubramaniam, K. S., Smaldone, L. A., \& Reger, B. 1999, ApJ, 510,422

Keller, C. U., Schüssler, M., Vögler, A., \& Zakharov, V. 2004, ApJ, 607, L59

Keppens, R. 2000, in Encyclopedia of Astronomy and Astrophysics, ed. P. Murdin (Bristol \& London: Institute of Physics \& Nature Publishing Group), 3193

Keppens, R., \& Martinez Pillet, V. 1996, A\&A, 316, 229

Khomenko, E. V., Martínez González, M. J., Collados, M., et al. 2005a, A\&A, 436, L27
Khomenko, E. V., Shelyag, S., Solanki, S. K., \& Vögler, A. 2005b, A\&A, 442, 1059

Knölker, M., \& Schüssler, M. 1988, A\&A, 202, 275

Kunasz, P. B., \& Auer, L. 1988, J. Quant. Spectr. Radiat. Transf., 39, 67

Leka, K. D., \& Skumanich, A. 1998, ApJ, 507, 454

Lites, B. W., Scharmer, G., Berger, T. E., \& Title, A. M. 2004, Sol. Phys., 221, 65

Martinez Pillet, V. 1997, in Advances in Physics of Sunspots, ed. B. Schmieder, J. Del Toro Iniesta, \& M. Vazquez (San Francisco: ASP), 212

Mathew, S. K., Martínez Pillet, V., Solanki, S. K., \& Krivova, N. A. 2007, A\&A, 465, 291

Muglach, K., Solanki, S. K., \& Livingston, W. C. 1994, in Solar Surface Magnetism, ed. R. J. Rutten, \& C. J. Schrijver (Dordrecht: Kluwer), 127 Nordlund, A. 1982, A\&A, 107, 1

Petrovay, K., \& Moreno-Insertis, F. 1997, ApJ, 485, 398

Pizzo, V. J. 1986, ApJ, 302, 785

Pizzo, V. J. 1990, ApJ, 365, 764

Rucklidge, A. M., Schmidt, H. U., \& Weiss, N. O. 1995, MNRAS, 273, 491

Sankarasubramanian, K., \& Rimmele, T. 2003, ApJ, 598, 689

Schüssler, M., Shelyag, S., Berdyugina, S., Vögler, A., \& Solanki, S. K. 2003, ApJ, 597, L173

Schüssler, M., \& Vögler, A. 2006, ApJ, 641, L73

Shelyag, S., Schüssler, M., Solanki, S. K., Berdyugina, S. V., \& Vögler, A. 2004, A\&A, 427, 335

Shelyag, S., Schüssler, M., Solanki, S. K., \& Vögler, A. 2007, A\&A, 469, 731

Simon, G. W., \& Weiss, N. O. 1970, Sol. Phys., 13, 85

Simon, G. W., Weiss, N. O., \& Nye, A. H. 1983, Sol. Phys., 87, 65

Sobotka, M., Vázquez, M., Bonet, J. A., Hanslmeier, A., \& Hirzberger, J. 1999, ApJ, 511, 436

Sobotka, M., Vázquez, M., Cuberes, M. S., Bonet, J. A., \& Hanslmeier, A. 2000, ApJ, 544, 1155

Solanki, S. K. 2003, A\&ARv, 11, 153

Spruit, H. C. 1976, Sol. Phys., 50, 269

Steiner, O. 2005, A\&A, 430, 691

Sütterlin, P. 1998, A\&A, 333, 305

Sütterlin, P., Schröter, E. H., \& Muglach, K. 1996, Sol. Phys., 164, 311

Vögler, A. 2003, Ph.D. Thesis, University of Göttingen, Germany, http://webdoc. sub.gwdg.de/diss/2004/voegler

Vögler, A. 2004a, A\&A, 421, 755

Vögler, A. 2004b, in Rev. Mod. Astron. 17: The Sun and Planetary Systems Paradigms for the Universe, ed. E. Schielicke (Weinheim, Germany: Wiley$\mathrm{VCH}), 69$

Vögler, A. 2005, Mem. Soc. Astron. It., 76, 842

Vögler, A., \& Schüssler, M. 2003, Astron. Nachr., 324, 399

Vögler, A., \& Schüssler, M. 2007, A\&A, 465, L43

Vögler, A., Shelyag, S., Schüssler, M., et al. 2003, in Modelling of Stellar Atmospheres, ed. N. E. Piskunov, W. W. Weiss, \& D. F. Gray (San Francisco: ASP), IAU Symp., 210, 157

Vögler, A., Bruls, J. H. M. J., \& Schüssler, M. 2004, A\&A, 421, 741

Vögler, A., Shelyag, S., Schüssler, M., et al. 2005, A\&A, 429, 335

Zirin, H., \& Wang, H. 1992, ApJ, 385, L27 